\title{
PÓ DE PEDRA: UMA ALTERNATIVA OU UM COMPLEMENTO AO USO DA AREIA NA ELABORAÇÃO DE MISTURAS DE CONCRETO?
}

\section{STONE POWDER: AN ALTERNATIVE OR AN ADDITION FOR THE USE OF SAND IN CONCRETE MIXTURES?}

\section{Rômulo Tadeu Menossi ${ }^{1}$, José Luiz Pinheiro Melges ${ }^{2}$, Jorge Luís Akasaki ${ }^{3}$, Jefferson Sidney Camacho ${ }^{4}$, João Victor Fazzan $^{5}$, Mauro Mitsuuchi Tashima ${ }^{6}$, Flávio Moreira Salles ${ }^{7}$}

${ }^{1}$ Mestre em Engenharia Civil, Universidade Estadual Paulista - UNESP - Campus de Ilha Solteira, e-mail: romulomenossi@hotmail.com

${ }^{2}$ Professor Doutor, Departamento de Engenharia Civil, Universidade Estadual Paulista - UNESP - Campus de Ilha Solteira, e-mail: jlmelges@dec.feis.unesp.br

${ }^{3}$ Professor Adjunto, Departamento de Engenharia Civil, Universidade Estadual

Paulista - UNESP - Campus de Ilha Solteira, e-mail: akasaki@dec.feis.unesp.br

${ }^{4}$ Professor Titular, Departamento de Engenharia Civil, Universidade Estadual

Paulista - UNESP - Campus de Ilha Solteira, e-mail: jsc@dec.feis.unesp.br

${ }^{5}$ Mestrando, Programa de Pós-Graduação em Engenharia Civil, Universidade Estadual Paulista - UNESP - Campus de Ilha Solteira, e-mail: jvfazzan@hotmail.com

${ }^{6}$ Doutorando, Instituto de Ciencia y Tecnología del Hormigón, Universidad Politécnica de Valencia - UPV, España, e-mail: tashima@japan.com

${ }^{7}$ Engenheiro, LCEC - Laboratório CESP de Engenharia Civil, Ilha Solteira, e-mail: flavio.salles@cesp.com.br

Alameda Bahia, 550 - Centro - 15385-000 - Ilha Solteira - SP - Fone: (18) 37431263.

\section{RESUMO}

Em função do elevado consumo de concreto, tanto em nível nacional, como em nível internacional, o uso de vários rejeitos industriais como matéria-prima para a sua elaboração tem dado destaque ao concreto como sendo um material ecologicamente compatível com o desenvolvimento sustentável. A maioria dos concretos é obtida a partir de misturas que utilizam agregados naturais, tendo-se, como exemplo, a areia natural e a brita. A retirada de areia de um rio pode agredir sua calha natural, levar a um aumento da vazão de água e acelerar o ritmo de erosão das margens. Por sua vez, o pó de pedra é um rejeito do processo de extração das britas, não possui destinação 
definida, e permanece estocado nos pátios das pedreiras formando enormes pilhas, alterando a paisagem e podendo provocar impactos ambientais, como, por exemplo, gerar poeira, obstruir canais de drenagem, e, quando carreados pela chuva, causar o assoreamento de rios. Além disso, também pode ser constatada a exaustão de reservas próximas aos grandes centros. Neste trabalho, apresentam-se resultados relativos à dosagem de concretos que tiveram, como agregados miúdos, areia natural e pó de pedra. Com o intuito de verificar a possibilidade de uso do pó de pedra, exatamente como se encontra nos pátios das pedreiras, não se descartou a fração inferior correspondente a $0,075 \mathrm{~mm}$. Constatou-se um aumento de resistência mecânica do concreto com pó de pedra em relação ao concreto com areia natural, principalmente para os traços mais pobres, embora também tenha sido observada uma diminuição da consistência, que por sua vez, pode ser compensada com o uso de aditivos plastificantes.

Palavras-chave: Impactos ambientais. Pó de pedra. Construção civil. Concreto.

\begin{abstract}
Due to the high consumption of concrete in the world, the use of several industrial residues as part of the raw materials used for its preparation has been giving distinction to the concrete as an ecologically compatible material with the sustainable development. Most mixtures using natural aggregates such as sand, gravel or crushed stone. Sand mining within river channels may modify its natural flume and increase its flow and the erosion of its banks. Besides, the stone powder is a rock crushing residue and it does not have a defined destination. It is stocked at the mines' stock yard, forming enormous hills that result in the alteration of natural habitats and causing environmental impacts, as, for example, generate dust, block drainage channels and when loaded by rain, causing the siltation of rivers due to aggradations. In addition, it can also be noted the exhaustion of the natural reserves near the big cities. In this paper some results are shown related to mixtures of concretes that have, as fine aggregates, natural sand and stone powder. With the objective to verify the influence of the stone powder use as it is found in the mines' stock yard, the particles passing through a $0,075 \mathrm{~mm}$ were not disregarded. It was noted that mixtures with stone powder had higher mechanical compressive strengths than mixtures with natural sand, but lesser slumps. However, in order to obtain concretes with better consistency it also can be used plasticizer additives.
\end{abstract}

Keywords: Environment impact. Stone powder. Civil construction. Concrete. 


\section{INTRODUÇÃO}

Em função do elevado consumo de concreto, tanto em nível nacional, como em nível internacional, a incorporação de vários rejeitos industriais, como, por exemplo, a sílica ativa, a cinza de casca de arroz, as fibras de borracha, as cinzas volantes, entre outros, tem dado destaque ao concreto como sendo um material ecologicamente compatível com o desenvolvimento sustentável. A grande maioria dos concretos é obtida a partir de misturas que utilizam agregados naturais, tendo-se, como exemplo, a areia natural e a brita.

Sabe-se que na produção de concreto armado, para cada metro cúbico de brita, são utilizados aproximadamente $2 \mathrm{~m}^{3}$ de areia natural. Sendo assim, a quantidade de areia consumida anualmente na construção civil brasileira é de 320 milhões de $\mathrm{m}^{3}$, volume este que daria para construir 7100 estádios como o Maracanã. Quase toda essa areia é retirada nas baixadas e leitos de rios (KUCK, 2003). Esses agregados, em volta das grandes cidades, estão ficando escassos, indicando a exaustão de reservas próximas aos grandes centros. Em São Paulo, por exemplo, a areia natural, em sua grande maioria, viaja distâncias superiores a $100 \mathrm{~km}$. Deste modo, tem-se um aumento do custo relacionado ao material, pois, além do aumento da distância de carga, pode-se mencionar ainda o incremento dos custos de transporte por pedagiamento e limites de peso transportados por eixo (JOHN, 2008). Por sua vez, o pó de pedra é um rejeito do processo de extração das britas, não possui destinação definida, e permanece estocado nos pátios das pedreiras formando enormes pilhas, alterando a paisagem e podendo gerar impactos ambientais, como, por exemplo, gerar poeira, obstruir canais de drenagem, e, quando carreados pela chuva, causar o assoreamento de rios (Figura 1).

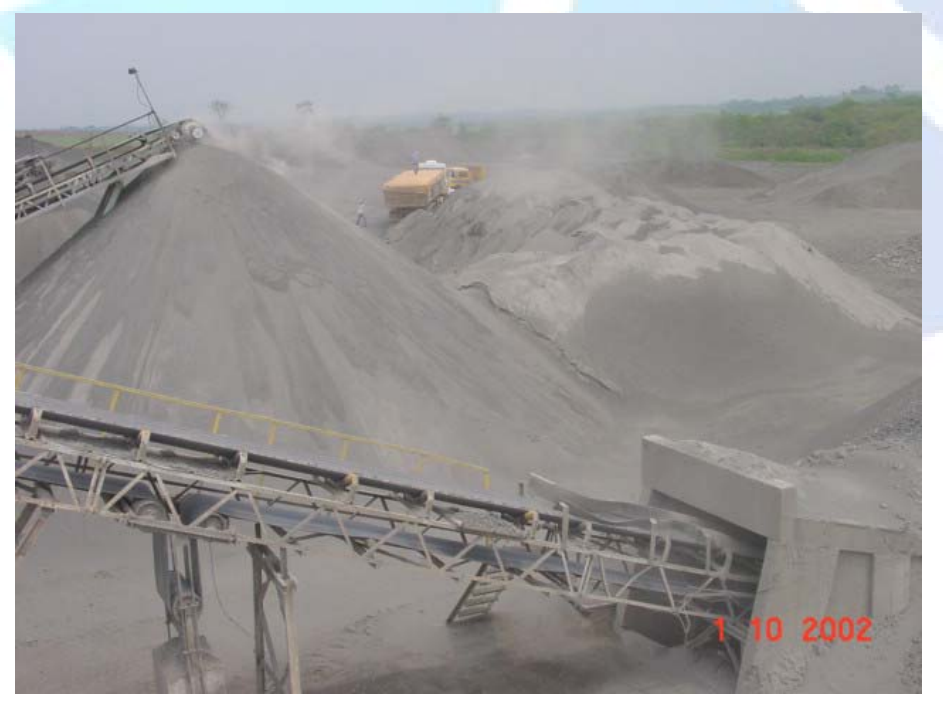

Figura 1. Grandes volumes estocados ao ar livre no pátio da Mineradora Fonte: Menossi (2004). 
Para aliviar esse impacto ambiental, o Centro de Tecnologia Mineral (CETEM) e a COPPE/UFRJ desenvolveram um projeto para obtenção de areia artificial com base em finos de pedreiras de brita. Esses finos possuem diferenças com relação à distribuição granulométrica, forma, textura e resistência mecânica das partículas, quando comparados com a areia natural, dificultando a trabalhabilidade do concreto. Para que eles possam ser empregados como agregados na construção civil, substituindo ou compondo com a areia natural, eles precisam passar por um processamento. Com a disponibilidade no mercado de novos equipamentos de britagem, que já conseguem produzir finos de formato adequado à produção de areia artificial, os pesquisadores puderam instalar em Matias Barbosa (MG) uma usinapiloto para produzi-la. Com isso, os finos acumulados nas pedreiras serão aproveitados como areia artificial, após tratamento com tecnologia limpa. A nova alternativa leva ainda à obtenção de uma areia com características físicas e químicas constantes, e diminui a quantidade de cimento para a preparação do concreto (KUCK, 2003).

Nessa mesma linha de ação, recentemente, duas unidades de pesquisa do Ministério da Ciência e Tecnologia - Instituto Nacional de Tecnologia (INT) e Centro de Tecnologia Mineral (CETEM) patentearam um processo que converte o pó fino resultante da exploração das rochas ornamentais em Santo Antônio de Pádua (RJ) em um dos principais insumos para a nova fábrica de argamassas que está sendo instalada naquele município. Deste modo, serão aproveitados mais de 16 mil toneladas/ano de resíduo sólido fino, que seriam lançados diretamente em mananciais da região, transformando um problema ambiental em matéria-prima para a indústria (BRASIL, 2003).

Segundo Kulaif (2001), a areia artificial produzida pelas pedreiras da Grande São Paulo torna-se competitiva pela proximidade destas (em torno de $35 \mathrm{~km}$ do centro de São Paulo) dos pontos de consumo, atingindo em 1999 uma participação da ordem de $7 \%$.

Neste estudo, apresentam-se resultados relativos à dosagem de concretos que tiveram, como agregados miúdos, areia natural e pó de pedra. Com o intuito de verificar a possibilidade de uso do pó de pedra exatamente como se encontra nos pátios das pedreiras, não se descartou a fração inferior correspondente a $0,075 \mathrm{~mm}$. Constatou-se um aumento de resistência mecânica do concreto com pó de pedra em relação ao concreto com areia natural, principalmente para os traços mais pobres, embora também tenha sido observada uma diminuição da consistência, que por sua vez, pode ser compensada com o uso de aditivos plastificantes.

\section{MATERIAL E MÉTODOS}

A metodologia empregada para definir composições de concreto e verificar suas características consistiu, primeiramente, em caracterizar os agregados. A seguir, 
foram desenvolvidos cinco traços de modo a se obter uma substituição gradual da areia natural por pó de pedra. O método de dosagem utilizado foi o Método IPT/EPUSP (HELENE e TERZIAN, 1993). O traço base produzido foi 1:3:3 em massa, onde foi feita a substituição da areia natural por pó de pedra na proporção de $25 \%, 50 \%, 75 \%$ e $100 \%$ em massa. O cimento utilizado foi o CP II 32 F. A Tabela 1 mostra os pontos básicos que diferenciam os cinco traços de concreto.

Inicialmente, foram adotadas uma relação a/c igual a 0,70 e uma consistência pelo abatimento do tronco de cone em $50 \pm 10 \mathrm{~mm}$. A seguir, foram obtidas as resistências à compressão aos 7, 28 e 91 dias, por meio de corpos de prova cilíndricos, de dimensões $100 \mathrm{~mm}$ x $200 \mathrm{~mm}$, para cada uma das misturas analisadas.

Tabela 1 - Traços produzidos para verificação do abatimento e relação a/c.

\begin{tabular}{c|c|c|c|c}
\hline Traço & Cimento & Areia (\%) & Pó-de-pedra (\%) & Brita (16mm) \\
\hline $\mathbf{1}$ & constante & 100 & 0 & constante \\
\hline $\mathbf{2}$ & constante & 75 & 25 & constante \\
\hline $\mathbf{3}$ & constante & 50 & 50 & constante \\
\hline $\mathbf{4}$ & constante & 25 & 75 & constante \\
\hline $\mathbf{5}$ & constante & 0 & 100 & constante \\
\hline
\end{tabular}

Posteriormente, utilizando-se as mesmas composições mencionadas na Tabela 1, fixou-se um abatimento de $60 \mathrm{~mm}$ e foram medidas a relação a/c utilizada para cada traço e a resistência à compressão dos corpos de prova aos 7, 28 e 91 dias. Esta análise foi feita para verificar a resposta do concreto, em termos de resistência (ordem de grandeza) e de rendimento (resistência dividida por consumo de cimento), com o aumento do fator $\mathrm{a} / \mathrm{c}$.

\section{RESULTADOS E DISCUSSÃO}

\subsection{Caracterização dos agregados}

A Associação Brasileira de Normas Técnicas, por meio da NBR 7211, define agregado miúdo cujos grãos passam pela peneira com abertura de malha de $4,75 \mathrm{~mm}$ e ficam retidos na peneira com abertura de malha de $150 \mu \mathrm{m}$ (ABNT, 2009).

Com base nas curvas granulométricas montadas para a areia e para o pó de pedra, concluiu-se que ambos puderam ser classificados como agregado miúdo. O pó de pedra possui origem basáltica, com aproximadamente $50 \%$ de sílica em sua composição química e apresenta cor cinza escuro. Também foi observado que o basalto que constitui o pó de pedra mostrou ter um comportamento inócuo na presença dos álcalis do cimento. A brita utilizada possui a mesma origem do pó de pedra em questão. 
A Tabela 2 mostra os resultados dos ensaios de caracterização realizados na areia natural, no pó de pedra e na brita.

Tabela 2 - Resultados das caracterizações realizadas na areia, no pó de pedra e no agregado graúdo.

\begin{tabular}{|c|c|c|c|}
\hline Ensaios & Areia Natural & Pó de Pedra & Brita \\
\hline Diâmetro Máximo $(\mathrm{mm})$ & 4,76 & 2,38 & 16 \\
\hline Módulo de Finura & 2,29 & 2,48 & 6,53 \\
\hline Massa Específica s.s.s. $\left(\mathrm{g} / \mathrm{cm}^{3}\right)$ & 2,655 & 2,787 & 2,789 \\
\hline Massa Específica Absoluta $\left(\mathrm{g} / \mathrm{cm}^{3}\right)$ & 2,644 & 2,751 & 2,745 \\
\hline Massa Unitária $\left(\mathrm{g} / \mathrm{cm}^{3}\right)$ & 1,520 & 1,491 & 1,551 \\
\hline Pulverulento $(\%)$ & 0,29 & 13,60 & 0,29 \\
\hline Absorção $(\%)$ & 0,39 & 2,71 & 1,90 \\
\hline Matéria Orgânica & Isento & Isento & Isento \\
\hline
\end{tabular}

Com base nos resultados apresentados na Tabela 2, foram observadas algumas diferenças entre a areia natural e o pó de pedra. O teor de material pulverulento do pó de pedra, obtido por meio das recomendações da NBR NM 46 (ABNT, 2003), mostrou-se acima do limite permitido pela NBR 7211 (ABNT, 2009) que fixa os valores máximos de material passante na peneira $0,075 \mathrm{~mm}$ em $3 \%$ para concretos submetidos a desgaste superficial e em 5\% para os demais concretos. Esses limites podem ser aumentados, respectivamente, para $10 \%$ e $12 \%$, desde que seja possível comprovar, por apreciação petrográfica realizada de acordo com a NBR 7389 (ABNT, 2009) que os grãos constituintes não interferem nas propriedades do concreto. Alguns estudos, no entanto, relatam que, para o pó de pedra, esse limite pode chegar em até $20 \%$, uma vez que o material pulverulento estaria colaborando para maior compacidade e, consequentemente, para maior durabilidade do concreto (REPORTAGEM, 2000). Além disso, levando-se em conta a questão ambiental, optou-se por não se aplicar qualquer recurso que pudesse diminuir a porcentagem do material pulverulento do pó de pedra. Deste modo, procurou-se utilizar o pó de pedra exatamente no estado em que se encontra nos pátios das pedreiras.

Foram realizados ensaios de acordo com a NBR NM 49 (ABNT, 2001) para verificar a presença de matéria orgânica nos agregados miúdos (areia e pó de pedra).

Outro ensaio de caracterização realizado com o pó de pedra foi o ensaio de perda de massa ao fogo. Neste ensaio, observou-se uma perda de aproximadamente $2,5 \%$ tanto para a temperatura de $600{ }^{\circ} \mathrm{C}$ como para a de $900{ }^{\circ} \mathrm{C}$, confirmando a ausência de matéria orgânica no material. 
3.2. Resultados de resistência à compressão e abatimento, com relação $\mathrm{a} / \mathrm{c}$ constante

A Tabela 3 fornece os valores de resistência à compressão axial alcançados pelos corpos de prova aos 7, 28 e 91 dias de idade, para cada um dos traços estudados. Nesta etapa do trabalho, fixou-se a relação a/c em 0,70 e a consistência pelo abatimento do tronco de cone em $50 \pm 10 \mathrm{~mm}$.

Tabela 3 - Valores de resistência à compressão aos 7, 28 e 91 dias.

\begin{tabular}{c|c|c|c|c}
\hline Traço & $\begin{array}{c}\text { Teor de } \\
\text { substituição }\end{array}$ & $\begin{array}{c}\text { Resistência aos } \\
\mathbf{7} \text { dias (MPa) }\end{array}$ & $\begin{array}{c}\text { Resistência aos } \\
\mathbf{2 8} \text { dias (MPa) }\end{array}$ & $\begin{array}{c}\text { Resistência aos } \\
\text { 91 dias (MPa) }\end{array}$ \\
\hline 1 & $0 \%$ & 16,8 & 18,8 & 22,0 \\
\hline 2 & $25 \%$ & 20,1 & 21,7 & 29,1 \\
\hline 3 & $50 \%$ & 20,8 & 24,0 & 33,1 \\
\hline 4 & $75 \%$ & 20,7 & 28,9 & 32,3 \\
\hline 5 & $100 \%$ & 22,0 & 31,2 & 37,4 \\
\hline
\end{tabular}

Para uma melhor comparação e entendimento dos valores apresentados na Tabela 3 a Figura 2 ilustra o agrupamento desses valores, sendo mais fácil identificar o crescimento de resistência ao longo do tempo.

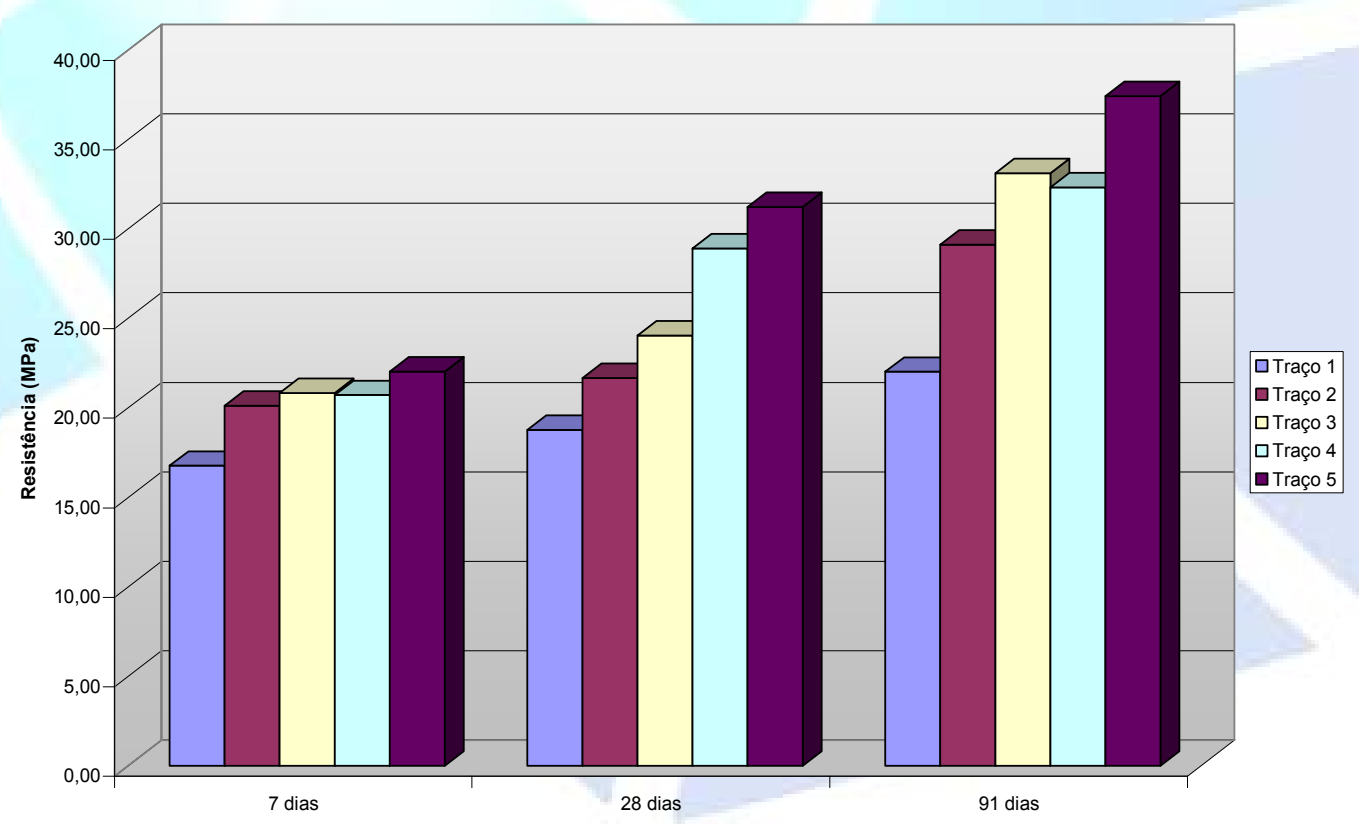

Figura 2. Resistência à compressão aos 7, 28 e 91 dias (a/c constante e igual a 0,7$)$.

Com base nos dados apresentados, pode-se observar que o traço 5, com $100 \%$ de pó de pedra, apresentou, em todas as idades, a maior resistência à compressão, embora também tenha apresentado o menor abatimento. Verifica-se que, quanto maior a porcentagem de pó de pedra no concreto, maior o ganho de resistência ao longo do tempo, se comparado ao concreto executado com areia natural. 
Comparando-se o traço 5, com $100 \%$ de adição de pó de pedra em substituição à areia natural, ao traço 1 , que utilizou apenas areia natural como agregado miúdo, observou-se um maior aumento de resistência com o tempo. Enquanto o traço 5 apresentou um aumento da resistência de aproximadamente $70 \%$ (de $22 \mathrm{MPa}$ aos 7 dias para $37,4 \mathrm{MPa}$ aos 91 dias), o traço 1 apresentou um aumento de 31\% (de 16,8 $\mathrm{MPa}$ aos 7 dias para $22 \mathrm{MPa}$ aos 91 dias). Já os traços 2, 3 e 4 apresentaram aumentos de, respectivamente, $45 \%, 59 \%$ e $56 \%$. Destaca-se que essas diferenças são significativas, uma vez que, dentro da faixa de consistência adotada, as quantidades de cimento, de água e da proporção de argamassa foram mantidas constantes. $\mathrm{Na}$ Figura 3, pode-se visualizar o acréscimo de resistência ao longo do tempo, para cada um dos traços.

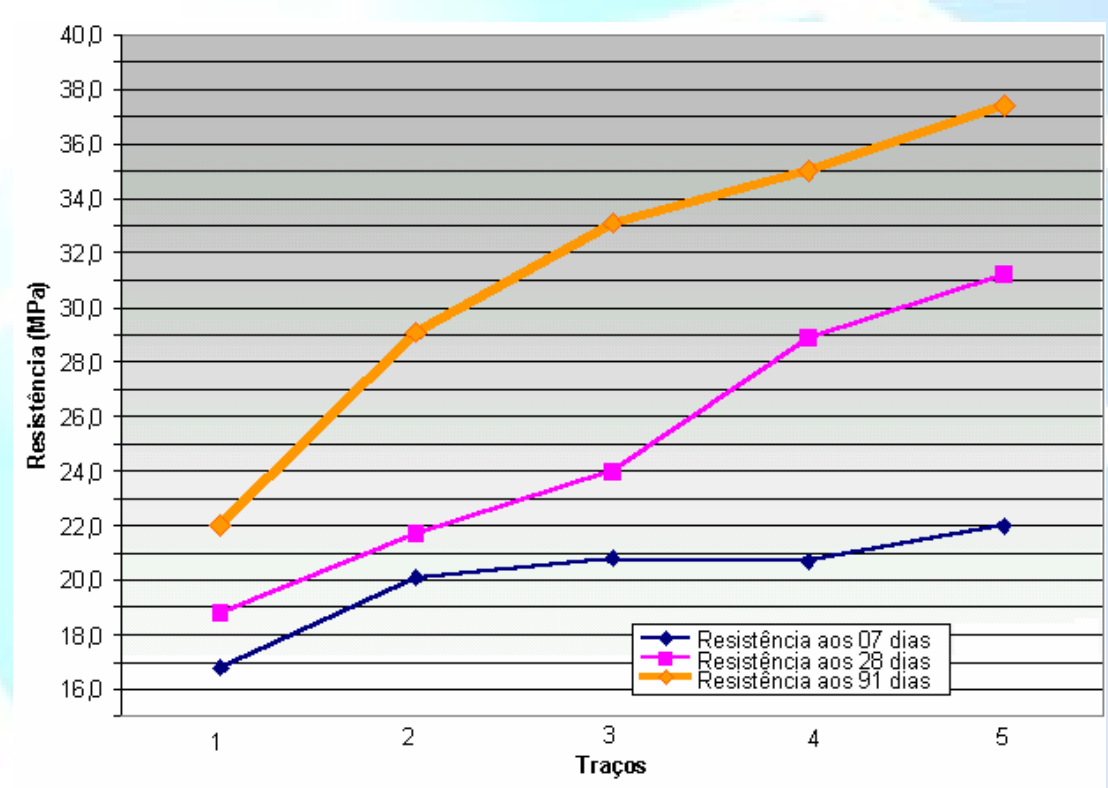

Figura 3. Comparação entre resistências aos 7, 28 e 91 dias

3.3. Resultados de resistência à compressão e da relação a/c, com abatimento constante

O objetivo principal desta análise foi verificar qual seria o efeito da substituição da areia natural pelo pó de pedra, em termos de resistência e de consumo de água, para um abatimento (slump) fixado em $60 \mathrm{~mm}$. A Figura 4 mostra os valores das relações a/c obtidas para cada um dos traços estudados. Observou-se um aumento na relação a/c com o aumento da proporção de pó de pedra no concreto. 


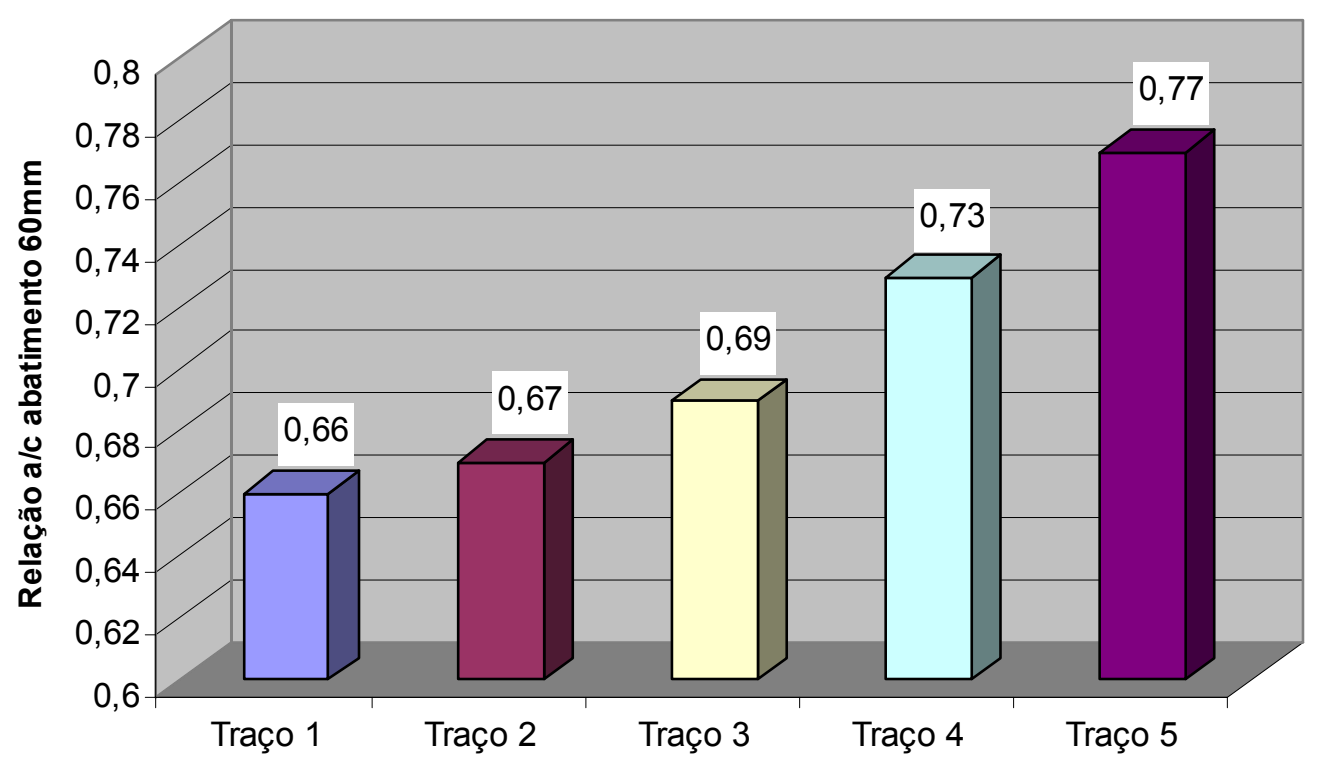

Figura 4. Comparação entre as diferentes relações a/c, para abatimento igual a $60 \mathrm{~mm}$

A Tabela 4 mostra os valores de resistência à compressão axial alcançados pelos corpos de prova aos 7, 28 e 91 dias, para cada um dos traços definidos. Para melhor visualização e entendimento dos valores mostrados na Tabela 4, pode-se observar, na Figura 5, o crescimento de resistência ao longo do tempo para cada um dos traços estudados.

Tabela 4 - Valores de resistência a compressão aos 7, 28 e 91 dias, para abatimento igual a 60 mm.

\begin{tabular}{c|c|c|c|c}
\hline Traço & $\begin{array}{c}\text { Teor de } \\
\text { Substituição }\end{array}$ & $\begin{array}{c}\text { Resistência aos } \\
\mathbf{7} \text { dias (MPa) }\end{array}$ & $\begin{array}{c}\text { Resistência aos } \\
\mathbf{2 8} \text { dias (MPa) }\end{array}$ & $\begin{array}{c}\text { Resistência aos } \\
\text { 91 dias (MPa) }\end{array}$ \\
\hline 1 & $0 \%$ & 16,7 & 22,8 & 25,1 \\
\hline 2 & $25 \%$ & 18,2 & 25,9 & 28,8 \\
\hline 3 & $50 \%$ & 20,4 & 31,1 & 34,5 \\
\hline 4 & $75 \%$ & 19,2 & 28,5 & 33,3 \\
\hline 5 & $100 \%$ & 19,5 & 28,6 & 31,7 \\
\hline
\end{tabular}




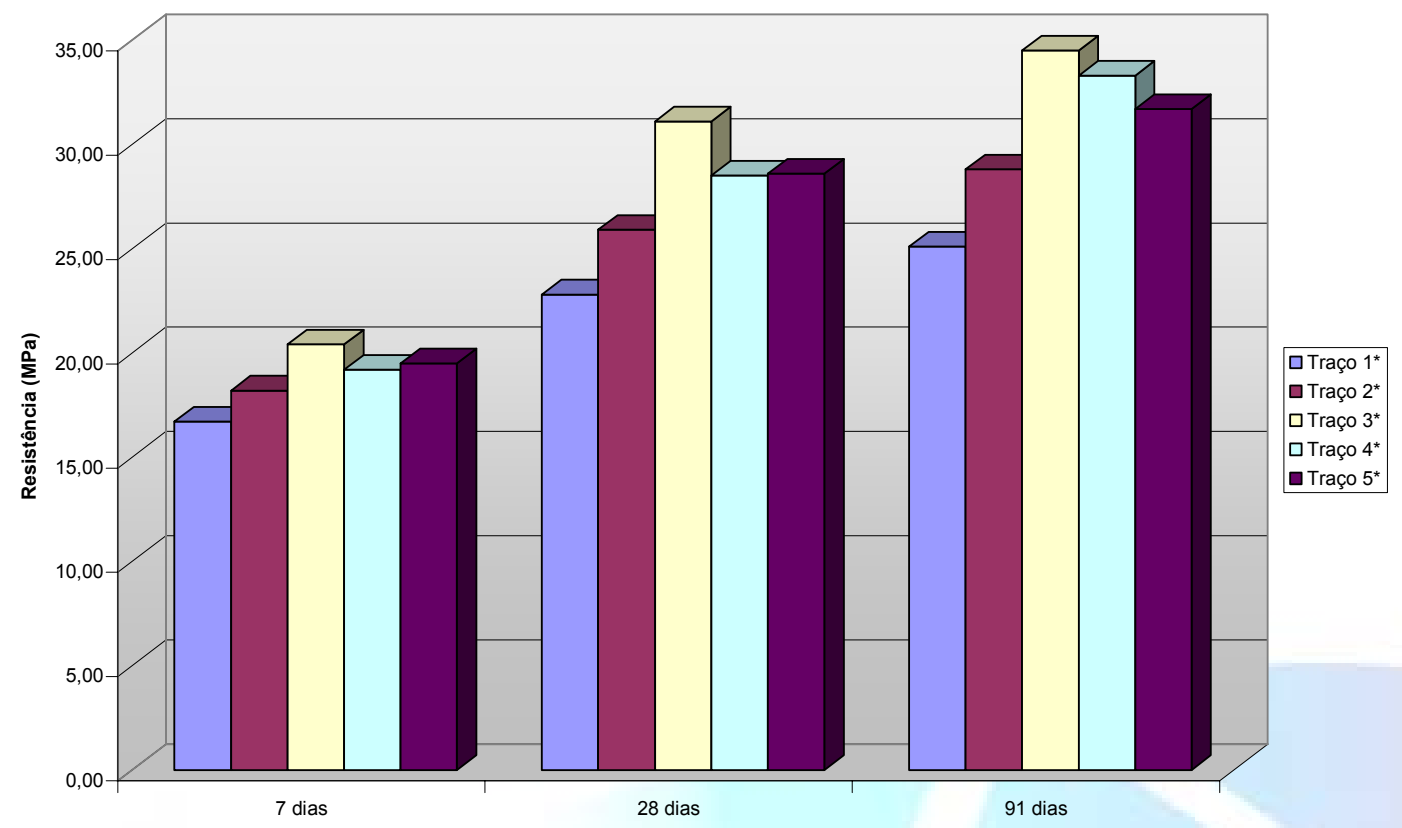

Figura 5. Resistência a compressão aos 7, 28 e 91 dias, para abatimento igual a $60 \mathrm{~mm}$

Comparando-se os traços 1 e 5, constatou-se que este fornece uma resistência maior que aquele, mesmo possuindo uma relação a/c maior. Observou-se, no entanto, que em todas as idades, as maiores resistências foram obtidas para o traço 3. Isto indica que a mistura admite uma substituição completa da areia pelo pó de pedra, sem perdas em termos de resistência e trabalhabilidade, embora o melhor comportamento esteja relacionado a uma substituição parcial de 50\%.

Tomando-se como referência as resistências obtidas para o traço 1, aos 7, 28 e 91 dias, percebe-se, também para esta análise, que o aumento da resistência observado para o traço 5 é maior que o aumento da resistência observado para o traço 1. Enquanto o traço 5 apresentou um aumento da resistência de aproximadamente $63 \%$ (de 19,5 $\mathrm{MPa}$ aos 7 dias para $31,7 \mathrm{MPa}$ aos 91 dias), o traço 1 apresentou um aumento de 50\% (de 16,7 MPa aos 7 dias para 25,1 MPa aos 91 dias). Já os traços 2, 3 e 4 apresentaram aumentos da resistência de, respectivamente, $58 \%, 69 \%$ e $73 \%$. $\mathrm{Na}$ Figura 6, tem-se o aumento da resistência à compressão, para cada um dos traços analisados, em relação ao tempo. 


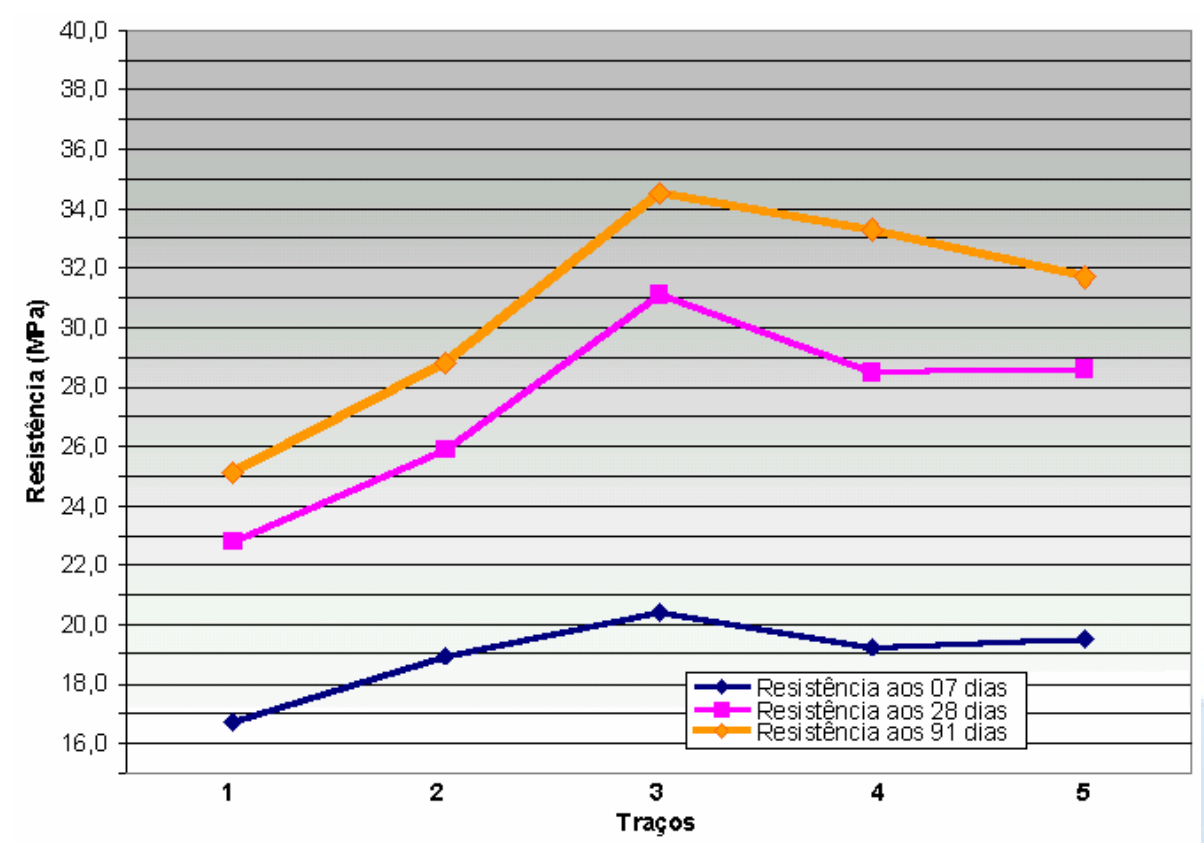

Figura 6. Resistência entre as resistências aos 7, 28 e 91 dias, para abatimento igual a $60 \mathrm{~mm}$

Os consumos relativos de cimento obtidos para os traços 1, 2, 3, 4 e 5 foram de, respectivamente, $307 \mathrm{~kg} / \mathrm{m}^{3}, 309 \mathrm{~kg} / \mathrm{m}^{3}, 310 \mathrm{~kg} / \mathrm{m}^{3}, 311 \mathrm{~kg} / \mathrm{m}^{3} \mathrm{e} 312 \mathrm{~kg} / \mathrm{m}^{3}$. Observou-se, deste modo, um pequeno aumento no consumo de cimento em função do aumento da porcentagem de substituição de areia por pó de pedra.

$\mathrm{Na}$ Tabela 5 e na Figura 7, têm-se, respectivamente, os valores e o gráfico do rendimento obtido para cada uma das misturas, aos 7, 28 e 91 dias. Um fato que se destaca é o rendimento apresentado pelo traço 3 , relativo à porcentagem de $50 \%$ de substituição de areia por pó de pedra, superior a 1,0, a partir dos 28 dias de idade.

Tabela 5 - Rendimento (em $\mathrm{kgf} / \mathrm{cm}^{2} / \mathrm{kg}$ de cimento).

\begin{tabular}{c|c|c|c}
\hline Traço & $\mathbf{0 7}$ dias & $\mathbf{2 8}$ dias & 91 dias \\
\hline 1 & 0,54 & 0,74 & 0,82 \\
\hline 2 & 0,59 & 0,84 & 0,93 \\
\hline 3 & 0,66 & 1,00 & 1,11 \\
\hline 4 & 0,62 & 0,92 & 1,07 \\
\hline 5 & 0,63 & 0,92 & 1,02 \\
\hline
\end{tabular}




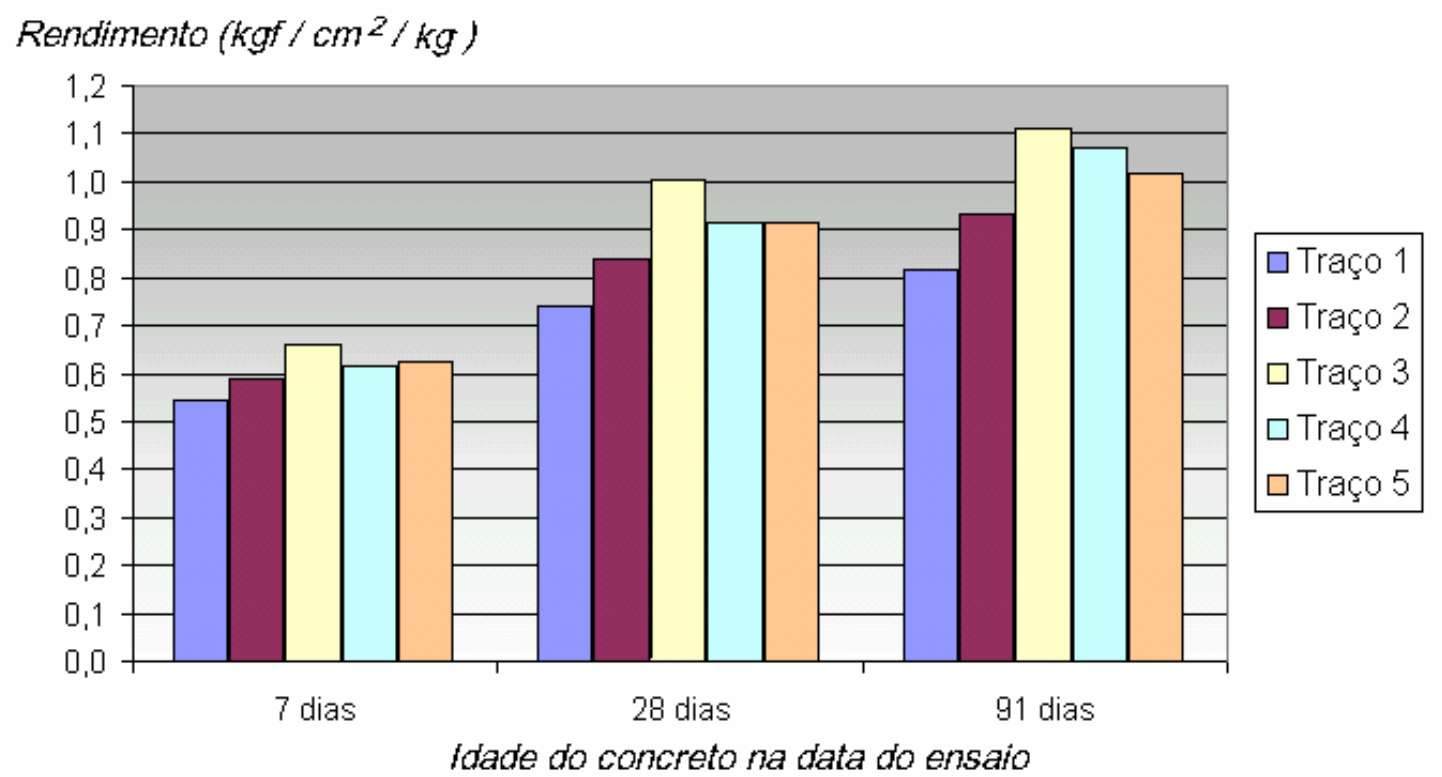

Figura 7. Rendimento (em $\mathrm{kfg} / \mathrm{cm}^{2} / \mathrm{kg}$ ).

\subsection{Análise dos Resultados}

Analisando-se os resultados obtidos, podem-se fazer as seguintes observações:

a) o pó de pedra analisado possui uma distribuição granulométrica que permite classificá-lo como agregado miúdo, de acordo com a NBR 7211 (ABNT, 2009) e mostrou ter comportamento inócuo na presença dos álcalis;

b) constatou-se um aumento de resistência mecânica do concreto com pó de pedra em relação ao concreto com areia natural, principalmente para os traços mais pobres, embora também tenha sido observada uma diminuição de trabalhabilidade, que por sua vez, pode ser compensada com o uso de aditivos plastificantes;

c) fixando-se um abatimento de $60 \mathrm{~mm}$, observou-se que a substituição de $50 \%$ da areia pelo pó de pedra resultou em um rendimento maior do que aqueles obtidos com $100 \%$ de areia ou com $100 \%$ de pó de pedra, sem perdas significativas de trabalhabilidade;

d) com relação à durabilidade, o material pulverulento presente no pó de pedra, em torno de $13 \%$, pode melhorar a característica do concreto com relação à compacidade, tornando-o menos permeável, mais coeso e trabalhável, resultando, portanto, em um concreto mais durável.

e) o consumo de cimento sofreu pequenos acréscimos em função do aumento da porcentagem de substituição de areia por pó de pedra.

\section{CONCLUSÕES}

É importante destacar que os resultados e conclusões apresentados neste trabalho referem-se a um determinado tipo de pó de pedra, com características 
específicas bem definidas, tendo sido obtido em uma determinada pedreira localizada na região noroeste do Estado de São Paulo.

Constatou-se que o pó de pedra pode ser considerado como um material alternativo ou complementar ao uso da areia natural. Embora o pó de pedra apresente condições para substituir a areia natural, mediante a aplicação de um aditivo para minimizar o efeito da perda de trabalhabilidade decorrente desta substituição, o ideal é que estes materiais sejam usados em conjunto, de modo a se obter vantagens dos pontos de vista ecológico, econômico e estrutural.

\section{AGRADECIMENTOS}

Os autores agradecem a Mineração Noroeste Paulista Ltda por investir em pesquisa tecnológica e financiamento de bolsa de mestrado e as agências de fomento CAPES, CNPq, FAPESP e ICITECH - Instituto de Ciencia y Tecnología del Hormigón da UPV - Universidad Politécnica de Valencia - Espanha, pelo apoio financeiro e científico para a realização das pesquisas do Grupo MAC - Materiais Alternativos de Construção da UNESP - Campus de Ilha Solteira.

\section{REFERÊNCIAS}

ABNT - ASSOCIAÇÃO BRASILEIRA DE NORMAS TÉCNICAS. NBR NM 46 Agregados - Determinação do material fino que passa através da peneira $75 \mu \mathrm{m}$, por lavagem. Rio de Janeiro, 2003.

ABNT - ASSOCIAÇÃO BRASILEIRA DE NORMAS TÉCNICAS. NBR NM 49 Agregado miúdo - Determinação de impurezas orgânicas. Rio de Janeiro, 2001.

ABNT - ASSOCIAÇÃO BRASILEIRA DE NORMAS TÉCNICAS. NBR 7211 Agregados para concreto - especificação. Rio de Janeiro, 2009.

ABNT - ASSOCIAÇÃO BRASILEIRA DE NORMAS TÉCNICAS. NBR 7389 Agregados - Análise petrográfica de agregado para concreto (parte 1: agregado miúdo). Rio de Janeiro, 2009.

REPORTAGEM. Areia Industrial Ganha Mercado. Minérios Extração \& Processamento, São Paulo, v. 20, n. 251, p.25-30, ago. 2000.

BRASIL. Ministério da Ciência e Tecnologia. Resíduo de rochas ornamentais vira insumo para uma nova fábrica. 2003. Disponível em: 
$<$ http://www.cetem.gov.br/noticias/cetem\%20midia/not_site mct 20_08_03_IV.html >. Acesso em: 30 de mai de 2011.

HELENE, P., TERZIAN, P. Manual de dosagem e controle de concreto. 2. ed. São Paulo: Editora Pini, 1993. 225 - 278 p.

JOHN, V. M. A construção, o meio ambiente e a reciclagem. PCC USP, São Paulo. 2008. Disponível em: $<$ http://www.reciclagem.pcc.usp.br/a_construcao_e.htm>. Acesso em: 30 de mai de 2011.

KUCK, D.W. Areia artificial reduz impacto ambiental de construção civil. 2003. Instituto Ciência Hoje. Disponível em: $<$ http://cienciahoje.uol.com.br/controlPanel/materia/view/3752>. Acesso em: $03 \mathrm{de}$ jul de 2008.

KULAIF, Y. Análise dos mercados de matérias-primas minerais: estudo de caso da indústria de pedras britadas do Estado de São Paulo. 2001. $144 \mathrm{f}$. Tese (Doutorado em Engenharia Mineral) - Escola Politécnica, Universidade de São Paulo, São Paulo. 2001.

MENOSSI, R.T. Utilização do pó de pedra basáltica em substituição à areia natural do concreto. 2004. 97 f. Dissertação (Mestrado em Engenharia Civil) Faculdade de Engenharia de Ilha Solteira, Universidade Estadual Paulista, Ilha Solteira. 2004. 